\title{
Relationship of Endothelial Shear Stress with Plaque Features with Coronary CT Angiography and Vasodilating Capability with PET
}

Kalykakis, Georgios-Eleftherios ; Antonopoulos, Alexios S ; Pitsargiotis, Thomas ; Siogkas, Panagiotis ; Exarchos, Themistoklis ; Kafouris, Pavlos ; Sakelarios, Antonis ; Liga, Riccardo ; Tzifa, Aphrodite ; Giannopoulos, Andreas ; Scholte, Arthur J H A ; Kaufmann, Philipp A ; Parodi, Oberdan ; Knuuti, Juhani ; Fotiadis, Dimitrios I ; Neglia, Danilo ; Anagnostopoulos, Constantinos D

\begin{abstract}
Background Advances in three-dimensional reconstruction techniques and computational fluid dynamics of coronary CT angiography (CCTA) data sets make feasible evaluation of endothelial shear stress (ESS) in the vessel wall. Purpose To investigate the relationship between CCTA-derived computational fluid dynamics metrics, anatomic and morphologic characteristics of coronary lesions, and their comparative performance in predicting impaired coronary vasodilating capability assessed by using PET myocardial perfusion imaging (MPI). Materials and Methods In this retrospective study, conducted between October 2019 and September 2020, coronary vessels in patients with stable chest pain and with intermediate probability of coronary artery disease who underwent both CCTA and PET MPI with oxygen 15-labeled water or nitrogen 13 ammonia and quantification of myocardial blood flow were analyzed. CCTA images were used in assessing stenosis severity, lesion-specific total plaque volume (PV), noncalcified PV, calcified PV, and plaque phenotype. PET MPI was used in assessing significant coronary stenosis. The predictive performance of the CCTA-derived parameters was evaluated by using area under the receiver operating characteristic curve (AUC) analysis. Results There were 92 coronary vessels evaluated in 53 patients (mean age, 65 years $\pm 7 ; 31$ men). ESS was higher in lesions with greater than $50 \%$ stenosis versus those without significant stenosis (mean, $15.1 \mathrm{~Pa} \pm 30$ vs $4.6 \mathrm{~Pa} \pm 4$ vs $3.3 \mathrm{~Pa} \pm 3 ; \mathrm{P}=$ .004). ESS was higher in functionally significant versus nonsignificant lesions (median, $7 \mathrm{~Pa}$ [interquartile range, 5-23 Pa] vs 2.6 Pa [interquartile range, 1.8-5 Pa], respectively; $\mathrm{P}$.001). Adding ESS to stenosis severity improved prediction (change in AUC, 0.10; 95\% CI: 0.04, 0.17; P =.002) for functionally significant lesions. Conclusion The combination of endothelial shear stress with coronary CT angiography (CCTA) stenosis severity improved prediction of an abnormal PET myocardial perfusion imaging result versus CCTA stenosis severity alone. (C) RSNA, 2021 Online supplemental material is available for this article. See also the editorial by Kusmirek and Wieben in this issue.
\end{abstract}

DOI: https://doi.org/10.1148/radiol.2021204381

Posted at the Zurich Open Repository and Archive, University of Zurich

ZORA URL: https://doi.org/10.5167/uzh-208807

Journal Article

Published Version

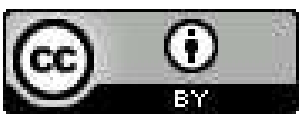

The following work is licensed under a Creative Commons: Attribution 4.0 International (CC BY 4.0) License. 
Originally published at:

Kalykakis, Georgios-Eleftherios; Antonopoulos, Alexios S; Pitsargiotis, Thomas; Siogkas, Panagiotis; Exarchos, Themistoklis; Kafouris, Pavlos; Sakelarios, Antonis; Liga, Riccardo; Tzifa, Aphrodite; Giannopoulos, Andreas; Scholte, Arthur J H A; Kaufmann, Philipp A; Parodi, Oberdan; Knuuti, Juhani; Fotiadis, Dimitrios I; Neglia, Danilo; Anagnostopoulos, Constantinos D (2021). Relationship of Endothelial Shear Stress with Plaque Features with Coronary CT Angiography and Vasodilating Capability with PET. Radiology, 300(3):549-556.

DOI: https://doi.org/10.1148/radiol.2021204381 


\title{
Relationship of Endothelial Shear Stress with Plaque Features with Coronary CT Angiography and Vasodilating Capability with PET
}

\author{
Georgios-Eleftherios Kalykakis, MSc* • Alexios S. Antonopoulos, MD, PhD* • Thomas Pitsargiotis, MSc • \\ Panagiotis Siogkas, $P h D$ - Themistoklis Exarchos, $P h D \cdot$ Pavlos Kafouris, MSc - Antonis Sakelarios, PhD • \\ Riccardo Liga, MD, PhD • Aphrodite Tzifa, MD, PhD • Andreas Giannopoulos, MD, PhD • \\ Arthur J. H. A. Scholte, MD, PhD - Philipp A. Kaufmann, MD, PhD - Oberdan Parodi, MD, PhD • \\ Juhani Knuuti, MD, PhD • Dimitrios I. Fotiadis, PhD • Danilo Neglia, MD, PhD • \\ Constantinos D. Anagnostopoulos, $M D, P h D$
}

From the Department of Informatics, Ionian University, Kerkyra, Greece (G.E.K., T.E.); Biomedical Research Foundation of the Academy of Athens, 4 Soranou Ephes-
siou St, 11527 Athens, Greece (G.E.K., T.P., P.K., C.D.A.); CMR Unit, Royal Brompton Hospital, London, England (A.S.A.); Department of Mechanical Engineering
and Aeronautics, University of Patras, Patras, Greece (T.P.); Department of Materials Science and Engineering University of Ioannina, Ioannina, Greece (P.S., D.I.F.);
Department of Informatics and Telecommunications, National and Kapodistrian University of Athens, Athens, Greece (P.K.); Biomedical Research Institute-FORTH,
Ioannina, Greece (A.S.); Cardiothoracic and Vascular Department, Azienda Ospedaliero Universitaria Pisana, Pisa, Italy (R.L.); Division of Imaging Sciences and Biomedi-
cal Engineering, King's College London, London, England (A.T.); Cardiac Imaging (P.A.K.) Department of Nuclear Medicine (A.G.), University Hospital Zurich, Zurich,
Switzerland (A.G.); Department of Cardiology, Heart Lung Center, Leiden University Medical Centre, Leiden, the Netherlands (A.J.H.A.S.); Institute of Clinical Physiol-
ogy, National Research Council-CNR, Pisa, Italy (O.P., D.N.); Institute of Information Science and Technologies, National Research Council-CNR, Pisa, Italy (O.P.);
PET Center, University Hospital and University of Turku, Turku, Finland (J.K.); Cardiovascular Department, Fondazione Toscana G. Monasterio, Pisa, Italy (D.N.); and
Sant'Anna School of Advanced Studies, Pisa, Italy (D.N.). Received December 8, 2020; revision requested January 25, 2021; revision received April 13; accepted May 10.
Address correspondence to C.D.A. (e-mail: cdanagnostopoulos@bioacademy.gr).

Study supported by the European Regional Development Fund, Operational Programme “Competitiveness, Entrepreneurship, and Innovation 2014-2020" (EPAnEK), titled "The Greek Research Infrastructure for Personalized Medicine (pMED-GR)" (grant number GR 5002802), by Greece and the European Union (European Social Fund-ESF) through the Operational Programme "Human Resources Development, Education and Lifelong Learning 2014-2020" in the context of the project "Assessment of coronary atherosclerosis: a new complete, anatomo-functional, morphological and biomechanical approach” (project number 504776) and by European Union's Horizon 2020 research and innovation program (grant number 689068, a SMARTool study).

${ }^{*}$ G.E.K. and A.S.A. contributed equally to this work.

Conflicts of interest are listed at the end of this article.

See also the editorial by Kusmirek and Wieben in this issue.

Radiology 2021; 300:549-556 • https://doi.org/10.1148/radiol.2021204381 • Content codes: CA CT

Background: Advances in three-dimensional reconstruction techniques and computational fluid dynamics of coronary CT angiography (CCTA) data sets make feasible evaluation of endothelial shear stress (ESS) in the vessel wall.

Purpose: To investigate the relationship between CCTA-derived computational fluid dynamics metrics, anatomic and morphologic characteristics of coronary lesions, and their comparative performance in predicting impaired coronary vasodilating capability assessed by using PET myocardial perfusion imaging (MPI).

\begin{abstract}
Materials and Methods: In this retrospective study, conducted between October 2019 and September 2020, coronary vessels in patients with stable chest pain and with intermediate probability of coronary artery disease who underwent both CCTA and PET MPI with oxygen 15-labeled water or nitrogen 13 ammonia and quantification of myocardial blood flow were analyzed. CCTA images were used in assessing stenosis severity, lesion-specific total plaque volume (PV), noncalcified PV, calcified PV, and plaque phenotype. PET MPI was used in assessing significant coronary stenosis. The predictive performance of the CCTA-derived parameters was evaluated by using area under the receiver operating characteristic curve (AUC) analysis.
\end{abstract}

Results: There were 92 coronary vessels evaluated in 53 patients (mean age, 65 years $\pm 7 ; 31$ men). ESS was higher in lesions with greater than $50 \%$ stenosis versus those without significant stenosis (mean, $15.1 \mathrm{~Pa} \pm 30$ vs $4.6 \mathrm{~Pa} \pm 4$ vs $3.3 \mathrm{~Pa} \pm 3 ; P=.004$ ). ESS was higher in functionally significant versus nonsignificant lesions (median, $7 \mathrm{~Pa}$ [interquartile range, 5-23 $\mathrm{Pa}$ ] vs $2.6 \mathrm{~Pa}$ [interquartile range, $1.8-5 \mathrm{~Pa}$ ], respectively; $P \leq .001$ ). Adding ESS to stenosis severity improved prediction (change in AUC, 0.10 ; 95\% CI: $0.04,0.17 ; P=.002$ ) for functionally significant lesions.

Conclusion: The combination of endothelial shear stress with coronary CT angiography (CCTA) stenosis severity improved prediction of an abnormal PET myocardial perfusion imaging result versus CCTA stenosis severity alone.

(C)RSA, 2021

Online supplemental material is available for this article.

Coronary CT angiography (CCTA) is a widely used noninvasive imaging modality for the assessment of coronary anatomy. Rapid technology improvements make the evaluation of phenotype and burden of coronary atherosclerotic plaques feasible and can improve the identification of flow-limiting lesions by providing information over and above that obtained by luminal stenosis severity alone $(1,2)$. Parallel to those developments, advances in computational fluid dynamics and three-dimensional reconstruction techniques allowed assessment of functional significance of coronary lesions by assessing CT fractional flow reserve or similar indexes (3). 


\begin{abstract}
Abbreviations
AUC $=$ area under the receiver operating characteristic curve, CCTA = coronary CT angiography, ESS = endothelial shear stress, EVINCI = Evaluation of Integrated Cardiac Imaging in Ischemic Heart Disease, MPI = myocardial perfusion imaging, PV = plaque volume
\end{abstract}

\section{Summary}

Coronary CT angiography-derived endothelial shear stress provided useful information for predicting impaired vasodilating capacity assessed by using PET myocardial perfusion imaging.

\section{Key Results}

- In a retrospective study of 92 coronary vessels in 53 patients with intermediate probability of coronary artery disease, coronary CT angiography-derived endothelial shear stress (ESS) was higher in lesions with greater than $50 \%$ stenosis $(P=.004)$ and in lesions associated with impaired myocardial blood flow measurements assessed by using PET (6.7 Pa vs $2.5 \mathrm{~Pa}$, respectively; $P \leq .001$ ).

- Adding ESS to stenosis severity improved prediction (improvement in area under the receiver operating characteristic curve, $0.10 ; P=.002)$ for flow-limiting lesions.

The evaluation of biomechanical forces in the vessel wall at CCTA is another possibility less well explored. Of particular interest is the assessment of endothelial shear stress (ESS), the product of dynamic viscosity and gradient of blood velocity near the arterial wall. ESS holds a key role in coronary plaque formation and progression because it disturbs the physiologic balance of the lumen wall (4-6). Also, increased blood flow at peak coronary vasodilation produces increases in local ESS, which is more pronounced in atherosclerotic lesions compared with normal vessels. This suggests an interplay between coronary atherosclerosis, vasodilating capability, and biomechanical forces (7).

The association of ESS to CCTA-derived plaque phenotype and coronary ischemia in the form of fractional flow reserve was previously explored (8). However, to our knowledge, no prior study has investigated the complex relationship between anatomic, morphologic, and biomechanical data with myocardial blood flow. The latter can be assessed accurately by using PET, which is the reference standard for such assessment. We hypothesized that ESS, which is sensitive to geometrical changes in the vessel wall, could be a possible predictor of impaired myocardial blood flow. The purpose of our study was therefore to assess the relationship between computational fluid dynamics metrics, anatomic and morphologic characteristics of coronary lesions, and their comparative performance in predicting impaired coronary vasodilating capability assessed by using PET myocardial perfusion imaging (MPI).

\section{Materials and Methods}

\section{Study Sample}

The Evaluation of Integrated Cardiac Imaging in Ischemic Heart Disease (EVINCI) was a prospective clinical European multicenter trial designed to assess the relative accuracy of commonly used imaging techniques in identifying patients with functionally significant coronary artery disease. It enrolled 475 patients who underwent a study of coronary anatomy by CCTA and at least one functional imaging test between March 23, 2009, and June 15, 2012 (9). An EVINCI and Simulation Modeling of Coronary Artery disease, or SMARTool, substudy has been subsequently performed to test the value of a CCTAbased fractional flow reserve surrogate index in detecting impaired coronary vasodilating capability (10). Our subanalysis was performed to assess the comparative performance of ESS, plaque volume (PV), and stenosis severity to predict abnormal PET MPI. Patients with stable chest pain and intermediate pretest likelihood were enrolled in the study. Exclusion criteria

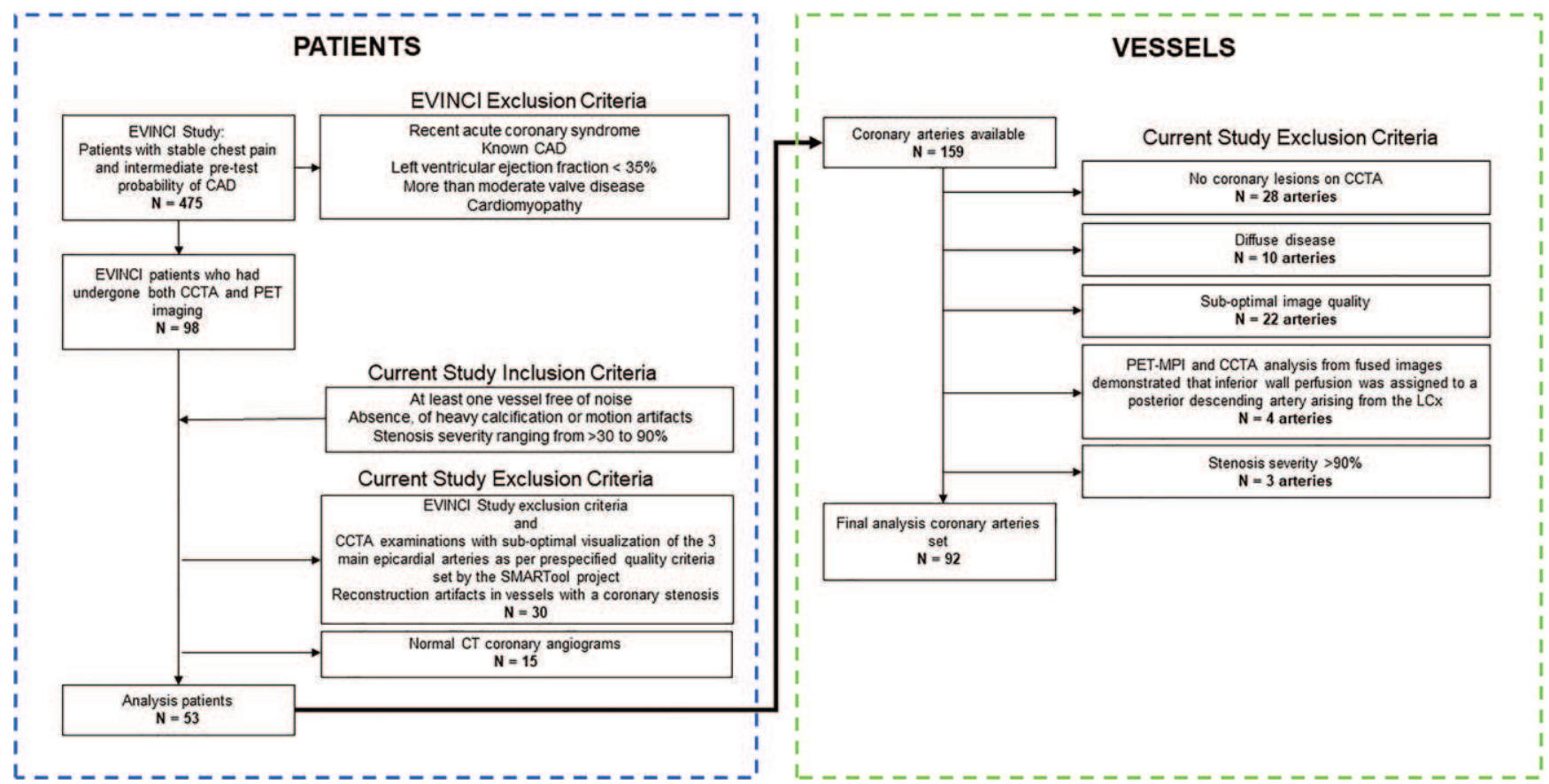

Figure 1: Study flow diagram of patients and vessels. CAD = coronary artery disease, $C C T A=$ coronary CT angiography, EVINCl = Evaluation of Integrated Cardiac Imaging in Ischemic Heart Disease, LCx = left circumflex, MPI = myocardial perfusion imaging, SMARTool = Simulation Modeling of Coronary Artery Disease. 
were recent acute coronary syndrome, known coronary artery disease, left ventricular ejection fraction less than 35\%, morethan-moderate valve disease, and cardiomyopathy. Details of inclusion and exclusion criteria are shown in Figure 1.

\section{CCTA Imaging and Data Analysis}

The CCTA images were obtained by using CT scanners that were 64 sections or more on the basis of the protocol of the EVINCI study by using an iodine-based contrast agent. Axial images were reconstructed with a section width less than $1.0 \mathrm{~mm}$ for most CCTA studies with a minimum section thickness of $0.5-0.6 \mathrm{~mm}$ to require maximum spatial resolution. Optimized reconstruction for the most suitable cardiac cycle was performed at diastole at $70 \%-80 \%$ of the R-R interval (Appendix E1 [online]) (9). The three-dimensional CCTA reconstruction proto- col was described in detail previously (11). Computational fluid dynamics were performed in the three main epicardial coronary arteries by using steady-state flow simulations for all vessels, assuming blood as Newtonian fluid, laminar flow, and rigid nonslip wall (12-14) (Appendix E1 [online]).

\section{Assessment of ESS}

The coordinates of the start and end of the lesion area on the basis of CCTA were identified by an independent reader (A.S.A., with $\geq 5$ years of experience in cardiac CT) with the SMARTool software (version 0.9.17) and with access only to CCTA images (15). The interval between readout sessions was 3 months, and the intraobserver coefficient of variation in a total of 40 of 92 (43\%) randomly selected vessels was $6.5 \%$. Total lesion length was divided into three segments of interest:
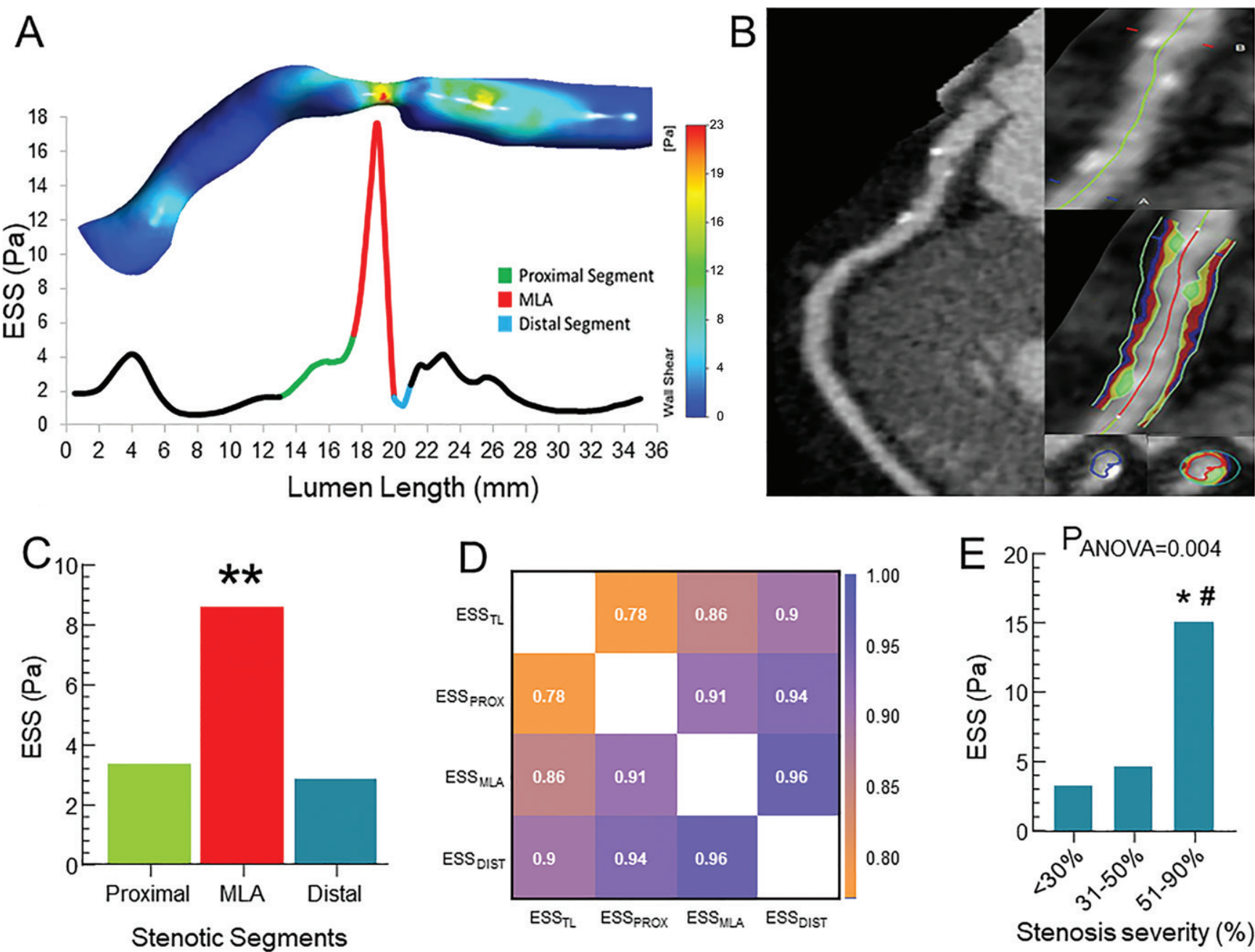

Figure 2: Endothelial shear stress (ESS) stenotic segments, plaque analysis, and stenosis severity. (A) Section of a right coronary artery of a 64 -year-old male patient with the calculated mean ESS segments $(0.5$-mm intervals) over the vessel length. Minimum lumen area (MLA) shows higher mean ESS values compared with the proximal and distal segments (mean, 10 Pa vs 3.6 Pa vs 1.8 Pa, respectively). (B) Left anterior descending artery plaque identification at coronary CT angiography (left panel) and curved planar reformatting (right upper panel); close-up at the volume of interest and color overlay for the plaque components (right middle panel; blue, necrotic core [-30 to $30 \mathrm{HU}$; ; red, fibrofatty [3 1-130 HU]; yellow, fibrous [1 31 -350 HU]; and green, dense calcium [>350 HU]). Cross-sectional view (right lower panel) shows the lumen boundaries with color overlay for individual plaque components. (C) Distribution of mean ESS values in the segmented lesions. ESS in proximal segments is higher compared with the distal ones. Minimum lumen area (MLA) displays the highest ESS values of the three calculated stenotic segments. ** $P<.05$ from proximal and distal. (D)

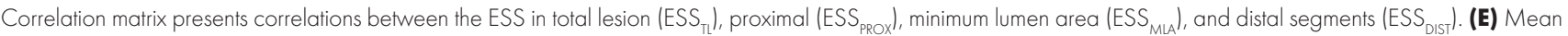
minimum lumen area ESS distribution over stenosis severity. $P_{\text {ANOVA }}=P$ value of the analysis of variance. ${ }^{*} P<.05$ versus stenosis severity $<30 \%$, \#P<.05 versus stenosis severity $31 \%-50 \%$ 
proximal, minimum lumen area $(\leq 3-\mathrm{mm}$ segment in total), and distal segment. The total lesion length and its three separate segments were individually analyzed for ESS assessment. It was averaged every $0.5-\mathrm{mm}$ interval along the total lesion length as well as the proximal, minimum lumen area, and distal segments of the stenosis, following the coronary centerline path (Fig 2A).

\section{Plaque Analysis}

Plaque analysis was performed on multiplanar reformatted images (average section thickness, 0.6; 0.6- $\mathrm{mm}$ increment) by an independent reader (A.S.A.) who had prior knowledge of the starting and ending lesion point and who was blinded to PET MPI results. The interval between readout sessions was 3 months, and the intraobserver coefficients of variation for total PV and noncalcified PV were $8.6 \%$ and $9.4 \%$, respectively. Total PV and the individual components were assessed at the total lesion length. Analysis was performed by using semiautomated software (Aquarius Workstation, version 4.4.13; TeraRecon) (Fig 2B) with manual corrections for wall and vessel territories where necessary. Quantified lesion-specific total PV was classified according to Hounsfield units into noncalcified PV $(-30$ to $350 \mathrm{HU})$ and calcified PV $(>350 \mathrm{HU})(16,17)$.

\section{PET Imaging and Data Analysis}

PET/CT imaging was performed in agreement with international guidelines and the EVINCI study protocol (18) by using oxygen 15-labeled water or nitrogen 13 ammonia. Details of PET imaging protocols and hybrid imaging analysis are in Appendix E1 (online) (19). PET was considered abnormal when more than one contiguous segment showed both stress myocardial blood flow of $2.3 \mathrm{~mL} / \mathrm{g} / \mathrm{min}$ or less and myocardial flow reserve of 2.5 or less for oxygen 15-labeled water water, or less than $1.79 \mathrm{~mL} / \mathrm{g} / \mathrm{min}$ and 2.0 or less for nitrogen $13 \mathrm{ammonia}$, respectively $(18,20)$.

\section{Statistical Analysis}

Continuous variables are presented as means \pm standard deviations or median and interquartile range, whereas qualitative variables are presented as absolute and relative frequencies. Normality was tested by using the Kolmogorov-Smirnov test. Probability values are two sided from the $t$ test and the Mann-Whitney $U$ test for continuous variables. Analysis of variance or Kruskal-Wallis test was selected for multiple group comparisons. $P<.05$ was considered to indicate statistical significance. The Spearman rank-correlation coefficient was selected to evaluate the relationship between ESS and plaque characteristics. The percentage composition of the individual plaque characteristics was defined as plaque component volume/total PV. An ESS threshold of 3.67 Pa, the median value of the total study sample at minimum lumen area, which is within the range of normal values quoted in the literature (21), was selected to classify low versus high ESS subgroups. The combined value of ESS, stenosis severity, and total PV (or noncalcified PV) for predicting an abnormal PET MPI result was evaluated by logistic regression models (clustered by patient) and calculation of the respective area under the receiver operating characteristic curve (AUC). Statistical analysis was

\begin{tabular}{lc} 
Table 1: Demographic and Clinical Study Sample \\
Characteristics \\
\hline Characteristic & Patients $(n=53)$ \\
\hline Mean age $(y)$ & $65 \pm 7[42-75]$ \\
No. of men & $31(58)$ \\
Mean age (y) & $64 \pm 8[42-75]$ \\
No. of women & $22(42)$ \\
Mean age $(y)$ & $66 \pm 5[59-73]$ \\
Symptom & \\
Typical angina & $8(15)$ \\
Atypical angina & $29(55)$ \\
Nonanginal chest pain & $16(30)$ \\
Risk factor & \\
Arterial hypertension & $28(53)$ \\
Diabetes mellitus & $9(17)$ \\
Obesity & $9(17)$ \\
Mean BMI (kg/m $\left.{ }^{2}\right)$ & $26.7 \pm 4$ \\
Medication & \\
\hline Oral antidiabetics & $7(13)$ \\
Insulin & $3(6)$ \\
\hline Statins & $37(70)$ \\
ACEi & $20(38)$ \\
Diuretics & $8(15)$ \\
ARBs & $8(15)$ \\
\hline blockers & $30(57)$ \\
Calcium antagonists & $8(15)$ \\
ASA & $43(81)$ \\
Nitrates & $9(17)$ \\
\hline & \\
\hline &
\end{tabular}

Note--Except where indicated, data are numbers of participants, with percentages in parentheses. Mean data are \pm standard deviation; data in brackets are range. $\mathrm{ACEi}=$ angiotensinconverting enzyme inhibitors, $\mathrm{ARB}=$ angiotensin II receptor blockers, ASA = acetylsalicylic acid, BMI = body mass index.

performed by using MedCalc Statistical Software (version 13; MedCalc Software) and Stata (version 13.0; StataCorp).

\section{Results}

\section{Patient and Vessel Characteristics}

Ninety-eight individuals from the EVINCI and SMARTool projects underwent PET MPI. Demographic, clinical, and coronary lesion characteristics of the total study sample are given in Tables 1 and 2. Thirty patients were excluded because of suboptimal image quality or reconstruction artifacts, and 15 patients were excluded because of normal CCTA. The remaining 53 patients (mean age, 65 years \pm 7 [standard deviation]; age range, $42-75$ years; 31 male patients [mean age, 64 years \pm 8 ]; 22 female patients [mean age, 66 years \pm 5 ]; $P=.21$ ) were evaluated for stenosis severity, plaque characteristics, and ESS. From a total of 159 coronary arteries, reasons for exclusion were as follows: 28 (two left anterior descending, 13 left circumflex, 13 right coronary arteries) did not have coronary lesions at CCTA, 10 demonstrated diffused disease (one left anterior descending, two left circumflex, seven right coronary arteries), 
22 had suboptimal image quality (six left anterior descending, 10 left circumflex, six right coronary arteries), PET MPI and CCTA analysis from fused images in four (right coronary arteries) demonstrated that inferior wall perfusion was assigned to a posterior descending artery from the left circumflex, and three (two left anterior descending, one right coronary artery) had a stenosis severity greater than $90 \%$. Subsequently, stenosis severity, plaque characteristics, and ESS were assessed in the remaining 92 coronary arteries (72 vessels with one stenosis per vessel).

\begin{tabular}{|c|c|}
\hline Characteristic & Vessel $(n=92)$ \\
\hline LAD & $44(48)$ \\
\hline $\mathrm{LCx}$ & $26(28)$ \\
\hline RCA & $22(24)$ \\
\hline Focal lesion vessels & $72(78)$ \\
\hline \multicolumn{2}{|l|}{ Stenosis severity } \\
\hline$<30 \%$ & $17(18)$ \\
\hline $31 \%-50 \%$ & $38(41)$ \\
\hline $51 \%-90 \%$ & $37(40)$ \\
\hline Mean lesion length (mm) & $14.1 \pm 9.7$ \\
\hline \multicolumn{2}{|l|}{ Mean plaque characteristics } \\
\hline Total plaque volume $\left(\mathrm{cm}^{3}\right)$ & $0.22 \pm 0.20$ \\
\hline Necrotic core $\left(\mathrm{cm}^{3}\right)$ & $0.04 \pm 0.04$ \\
\hline Fibrofatty tissue $\left(\mathrm{cm}^{3}\right)$ & $0.08 \pm 0.07$ \\
\hline Fibrous tissue $\left(\mathrm{cm}^{3}\right)$ & $0.08 \pm 0.07$ \\
\hline Calcified plaque $\left(\mathrm{cm}^{3}\right)$ & $0.03 \pm 0.05$ \\
\hline Noncalcified plaque $\left(\mathrm{cm}^{3}\right)$ & $0.19 \pm 0.17$ \\
\hline Outer wall volume $\left(\mathrm{cm}^{3}\right)$ & $0.37 \pm 0.33$ \\
\hline Lumen volume $\left(\mathrm{cm}^{3}\right)$ & $0.11 \pm 0.10$ \\
\hline \multicolumn{2}{|l|}{ Mean ESS segment } \\
\hline ESS TL $(\mathrm{Pa})$ & $4.1 \pm 7.8$ \\
\hline ESS proximal $(\mathrm{Pa})$ & $3.3 \pm 5.6$ \\
\hline ESS MLA (Pa) & $8.6 \pm 19.7$ \\
\hline ESS distal $(\mathrm{Pa})$ & $2.9 \pm 5.2$ \\
\hline \multicolumn{2}{|c|}{$\begin{array}{l}\text { Note.-Except where indicated, data are numbers of vessels, with } \\
\text { percentages in parentheses. Mean data are } \pm \text { standard deviation. } \\
\text { ESS = endothelial shear stress, LAD = left anterior descending, } \\
\text { LCx = left circumflex, MLA = minimum lumen area, RCA = } \\
\text { right coronary artery, TL = total lesion. }\end{array}$} \\
\hline
\end{tabular}

In terms of patients, 22 of 53 (42\%) had single-vessel disease, 23 of $53(43 \%)$ had two-vessel disease, and eight of 53 (15\%) had three-vessel disease.

\section{ESS Distribution across Coronary Lesions and Relationship with Stenosis Severity}

We used the ESS at minimum lumen area as the surrogate marker for lesion-related ESS in all the analyses because ESS indexes were highly intercorrelated $(r>0.78$ for all comparisons) (Fig 2D). ESS at minimum lumen area was higher compared with proximal ESS (mean, 8.6 $\mathrm{Pa} \pm 20$ vs 3.4 $\mathrm{Pa} \pm 5.6$, respectively; $P<.001)$ and distal ESS $(8.6 \mathrm{~Pa} \pm 20$ vs $2.9 \mathrm{~Pa}$ \pm 5.2 , respectively; $P<.001$ ) (Fig $2 \mathrm{C}$ ). ESS was higher in lesions with greater than $50 \%$ stenosis compared with segments with lesions with less than $50 \%$ stenosis (mean, $15.1 \mathrm{~Pa} \pm 30$ vs $4.6 \mathrm{~Pa} \pm 4$ vs $3.3 \mathrm{~Pa} \pm 3$, respectively; $P=.004$ ) (Fig $2 \mathrm{E}$, Table E1 [online]). The mean total time for ESS calculation was 20 minutes \pm 10 .

\section{Relationship between ESS, PV, and Plaque Composition}

There was a weak positive correlation between ESS and total PV $(r=0.28 ; P=.007)$ and between ESS and noncalcified PV $(r=0.27 ; P=.10)($ Fig 3A, 3B). In examining the relationship between ESS with plaque phenotype, we observed a weak positive correlation between ESS and the individual components of noncalcified PV (ie, necrotic core and fibrous and fibrofatty plaque volume; $r=0.22-0.25 ; P<.05$ ) (Fig 3C). Noncalcified PV was higher in vessels with high ESS (median, 0.17 $\mathrm{cm}^{3}$ [interquartile range, $0.12-0.29 \mathrm{~cm}^{3}$ ] vs $0.11 \mathrm{~cm}^{3}$ [interquartile range, $0.07-0.17 \mathrm{~cm}^{3}$ ], respectively; $P=.002$ ) (Table E2 [online]), but there was no difference in plaque composition (necrotic core, fibrous, fibrofatty, and calcified plaque, $P=$.12-.64) (Fig E1 [online]).

\section{Relationship between ESS, Stenosis Severity, PV, and Quantitative PET Perfusion}

Of the total 92 vessels analyzed, 46 of 92 (50\%) had both normal stress myocardial blood flow and myocardial flow reserve, whereas both parameters were abnormal in 23 of 92 (25\%) vessels. ESS was higher in vessels with abnormal myocardial blood flow and myocardial flow reserve (Fig 4A) (Table E3 [online]).
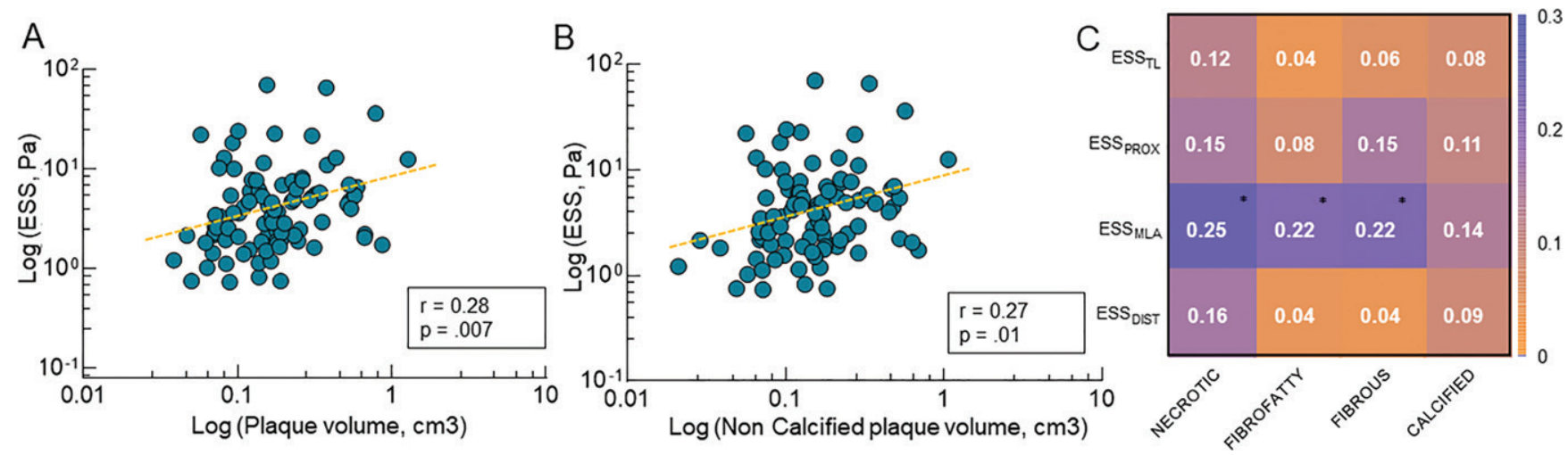

Figure 3: (A-C) Correlations between endothelial shear stress (ESS) and plaque characteristics. (A, B) Scatterplots show Spearman $r$ correlation between ESS and plaque volume (A) and noncalcified plaque volume (B). (C) Correlation matrix shows the correlations between ESS and plaque characteristics. ${ }^{\star} P<.05$. ESS ${ }_{\text {DIST }}=$ distal $\mathrm{ESS}, \mathrm{ESS}_{\mathrm{MLA}}=\mathrm{ESS}$ minimum lumen area, $\mathrm{ESS}_{\mathrm{PROX}}=$ proximal $\mathrm{ESS}, \mathrm{ESS}_{\mathrm{TL}}=\mathrm{ESS}_{\text {total lesion }}$ 
When the analyzed vessels were stratified according to subgroups of stenosis severity (below or above 50\%) and ESS (high vs low), five of $23(22 \%)$ of vessels with stenosis severity less than $50 \%$ and high ESS showed an abnormal PET MPI result (Fig 4B, Fig E2 [online]). Lesion-specific PV; noncalcified PV; and plaque characteristics such as calcium, fibrotic content, necrotic core, and fibrofatty plaque volume were not predictors of an abnormal PET MPI examination (AUC, 0.59-0.63; $P=.08-.63$ ). The combination of those parameters with stenosis severity did not add incremental value to the model (AUC, 0.74-0.77; $P$ $=.22-.68)$ (Table E4 [online]). However, both stenosis severity and ESS were predictors of impaired vasodilating capability
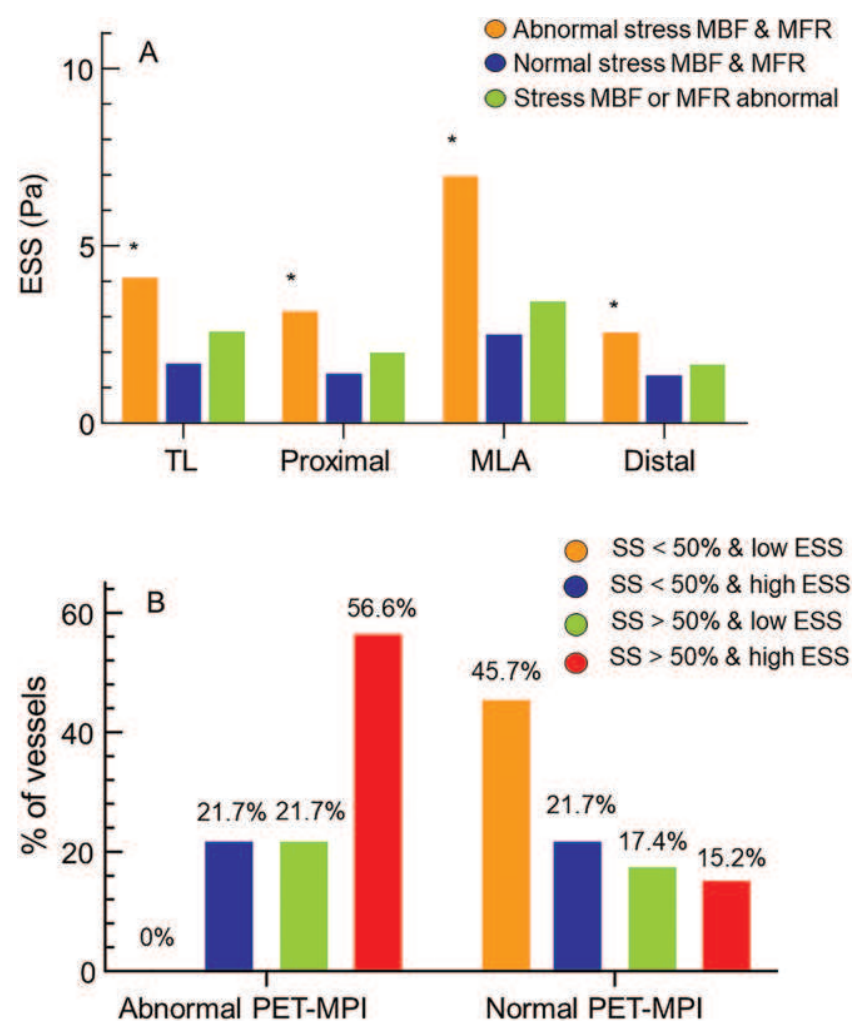

Figure 4: Distribution of endothelial shear stress (ESS) in vessels with normal and abnormal PET myocardial perfusion imaging (MPI) result. (A) Median ESS for total lesion (TL) and for each individual stenotic segment in vessels with normal versus abnormal PET MPI. * $P<.05 . \mathrm{MBF}=$ myocardial blood flow, MFR = myocardial flow reserve, $M L A=$ minimum lumen area. (B) Percentage of vessels with normal or abnormal PET MPI across strata of ESS and stenosis severity (SS).
(AUC, $0.73-0.79 ; P<.001$ for both) (Fig E3A, E3B [online]). The optimal threshold of ESS as a predictor was $5.4 \mathrm{~Pa}$, with sensitivity of 17 of 23 (74\%; 95\% CI: 52, 90) and specificity of 37 of 46 (80\%; 95\% CI: 66, 91), respectively. Importantly, addition of ESS to CCTA stenosis severity provided incremental value in predicting an abnormal PET MPI result (change in AUC, 0.10; 95\% CI: 0.04, 0.17; $P=.002$ ) (Table 3) (Fig E3C [online]).

\section{Discussion}

Advances in three-dimensional reconstruction techniques and computational fluid dynamics of cardiac CT angiography (CCTA) data sets make feasible the evaluation of endothelial shear stress (ESS) in the coronary artery wall. Our study was designed to investigate the relationship between ESS, stenosis severity, lesion-specific plaque volume (PV), and phenotype and their comparative performance in predicting impaired coronary vasodilating capability assessed by using PET myocardial perfusion imaging (MPI). Our main findings are as follows: CCTAderived ESS is higher in the proximal and minimum lumen area segments of a coronary lesion (mean proximal ESS, 3.4 Pa \pm 5.6; mean ESS minimum lumen area, 8.6 $\mathrm{Pa} \pm 19.7$; mean distal ESS, $2.9 \mathrm{~Pa} \pm 5.2 ; P<.001)$, there is a weak positive correlation between ESS and the individual components of noncalcified PV $(r=0.22-0.25 ; P<.05)$, and ESS has incremental value when combined with stenosis severity in classifying flowlimiting lesions assessed by using PET MPI (change in area under the receiver operating characteristic curve, $0.10 ; 95 \% \mathrm{CI}$ : $0.04,0.17 ; P=.002$ ).

The highest ESS values were observed at the minimum lumen area of a coronary lesion, whereas the lowest ESS values were observed at the distal plaque segment $(P<.001)$. This is an expected finding because plaque anatomy alters the blood flow and creates downstream flow recirculation zones that result in low ESS regions $(13,22-24)$. ESS is increased in segments with stenosis severity greater than $50 \%$ (mean, $15.1 \mathrm{~Pa} \pm 29.8$ vs 4.6 $\mathrm{Pa} \pm 4$ vs $3.3 \mathrm{~Pa} \pm 3$, respectively; $P=.004$ ), which also agrees with previous work (8).

Low ESS was previously associated with a more vulnerable plaque phenotype $(25,26)$ and a higher percentage of necrotic core (26). Evidence $(22,27)$ also suggests that high ESS is linked to high-risk plaques in coronary arteries. These findings may be explained by numerous factors: the different values used in the various studies to define high ESS $(22,26)$, the possibility

\section{Table 3: Diagnostic Value of ESS when Combined with Cardiac CT Angiography Anatomic Information for an Abnormal PET Myocardial Perfusion Imaging Result}

\begin{tabular}{llll}
\hline Variable & AUC & Change in AUC & $P$ Value \\
\hline Stenosis severity $>50 \%$ & $0.73(0.61,0.83)$ & & $<.001$ \\
ESS & $0.79(0.68,0.88)$ & $0.10^{*}(0.04,0.17)$ & $<.001$ \\
Stenosis severity $>50 \%$ with ESS & $0.83(0.72,0.91)$ & $0.03^{*}(-0.04,0.11)$ & $.002^{*}$ \\
Stenosis severity $>50 \%$ with TPV & $0.76(0.64,0.86)$ & $0.07^{\dagger}(-0.001,0.14)$ & $.38^{*}$ \\
Stenosis severity $>50 \%$ with TPV and ESS & $0.83(0.72,0.91)$ & $.054^{\dagger}$ \\
\hline
\end{tabular}

Note.-Data in parentheses are 95\% CIs. AUC = area under the curve, ESS = endothelial shear stress, TPV = total plaque volume.

* Difference from stenosis severity greater than $50 \%$.

${ }^{\dagger}$ Difference from stenosis severity greater than $50 \%$ combined with total plaque volume. 
of a U-shaped association between ESS and plaque vulnerability (22), the uncertainty over the physiologic ESS values in human coronary circulation $(5,28-31)$, and the study sample (ie, healthy vessels or vessels with established atherosclerosis). In our study, ESS was positively correlated with total PV and noncalcified PV $(r=0.27-0.28 ; P \leq .01)$. Alterations in ESS may relate to changes in endothelial mechanical transduction, known as mechanotransduction, and subsequent endothelium and plaque biology changes associated with plaque vulnerability. These would manifest as an increase in the volume of noncalcified plaque. ESS was higher in vessels with an abnormal stress myocardial blood flow and myocardial flow reserve (median, 6.9 $\mathrm{Pa}$ [interquartile range, 5-22.6 Pa] vs $2.5 \mathrm{~Pa}$ [interquartile range, 1.8-4.9 Pa], respectively; $P<.001$ ). In addition, stenosis severity and lesion-specific ESS were both predictors of impaired vasodilating capability $(P<.001$ for both). There is a potential explanation for the predictive ability of ESS. ESS may increase in endothelial dysfunction with noncalcific plaques as the level of stenosis increases because of impaired mechanical transduction. This condition would translate into reduced capability of dilatation. An additional explanation could be that chronic increase in ESS and, therefore, of vasodilating stimulus could exhaust the already impaired endothelial mediated flow reserve (32). However, total PV, noncalcified PV, and the individual plaque components such as calcium and fibrotic content were not associated with impaired vasodilation. Our results are similar to those previously reported (33) but also differ from results in other studies $(2,34)$. There are two possible explanations for the discrepant observations in the literature: methodologic differences in the extent of the arterial segment analyzed and reference standards used (fractional flow reserve or PET/SPECT). Our findings suggest that ESS is hemodynamically relevant and may partly explain the functional consequences of coronary plaque anatomy and geometry within a lumen. For example, nonobstructive coronary lesions may be functionally significant depending on plaque geometry and anatomy, of which ESS is a sensitive marker.

Our study had limitations. We did not assess the impact of side branches on ESS and we did not study ESS in hyperemic conditions, which could provide additional information regarding the relationship of ESS with PET MPI. In agreement with previous studies, in vessels with multiple stenoses only the most important lesion was selected for analysis $(23,35)$. A number of patients and vessels had to be excluded from our analysis because ESS, unlike other fluid dynamic parameters (ie, pressure), can be affected by image motion and quality of segmentation. Finally, in agreement with previous studies (26), we selected for analysis only the plaque phenotype without assessing other vessel characteristics (eg, positive remodeling, napkin ring, and spotty calcification) because this was beyond the aims of our study. ESS has incremental value when combined with stenosis severity in classifying flow-limiting lesions as assessed by using PET MPI, although these findings do not necessarily support the value of hybrid imaging in every patient.

In conclusion, endothelial shear stress (ESS) was positively correlated with the volume of coronary plaques and luminal stenosis severity. In addition, plaque anatomy and the resulting changes in fluid dynamics captured by ESS seem to be important informants of the functional significance of a coronary lesion. Moreover, ESS can add incremental value to stenosis severity by improving prediction of impaired vasodilating capacity assessed by using PET myocardial perfusion imaging.

Acknowledgment: The authors thank Dr George Benetos for his insightful comments and advice in statistical analysis.

Author contributions: Guarantors of integrity of entire study, P.S., P.K., A.S., C.D.A.; study concepts/study design or data acquisition or data analysis/interpretation, all authors; manuscript drafting or manuscript revision for important intellectual content, all authors; approval of final version of submitted manuscript, all authors; agrees to ensure any questions related to the work are appropriately resolved, all authors; literature research, G.E.K., T.P., T.E., A.S., A.T., A.G., P.A.K., O.P., D.I.F., C.D.A.; clinical studies, G.E.K., T.P., R.L., A.G., P.A.K., O.P., J.K., D.N., C.D.A.; experimental studies, T.P., P.S., R.L.; statistical analysis, G.E.K., A.S.A., T.P., P.S., T.E., P.K., A.S., A.T., A.G., D.I.F., C.D.A.; and manuscript editing, all authors

Disclosures of Conflicts of Interest: G.E.K. disclosed no relevant relationships. A.S.A. disclosed no relevant relationships. T.P. disclosed no relevant relationships. P.S. disclosed no relevant relationships. T.E. disclosed no relevant relationships. P.K. disclosed no relevant relationships. A.S. disclosed no relevant relationships. R.L. disclosed no relevant relationships. A.T. disclosed no relevant relationships. A.G. disclosed no relevant relationships. A.J.H.A.S. disclosed no relevant relationships. P.A.K. Activities related to the present article: disclosed no relevant relationships. Activities not related to the present article: disclosed money to author for consultancy from GE Healthcare; grants/grants pending from GE Healthcare. Other relationships: disclosed no relevant relationships. O.P. disclosed no relevant relationships. J.K. Activities related to the present article: disclosed no relevant relationships. Activities not related to the present article: disclosed money to author for consultancies from GE Healthcare, AstraZeneca; payment for lectures from Lundbeck, Bayer, Merck, BoehringerIngelheim. Other relationships: disclosed no relevant relationships. D.I.F. disclosed no relevant relationships. D.N. disclosed no relevant relationships. C.D.A. disclosed no relevant relationships.

\section{References}

1. Park HB, Heo R, Ó Hartaigh B, et al. Atherosclerotic plaque characteristics by $\mathrm{CT}$ angiography identify coronary lesions that cause ischemia: a direct comparison to fractional flow reserve. JACC Cardiovasc Imaging 2015;8(1):1-10.

2. Gaur S, Øvrehus KA, Dey D, et al. Coronary plaque quantification and fractional flow reserve by coronary computed tomography angiography identify ischaemia-causing lesions. Eur Heart J 2016;37(15):12201227

3. Nørgaard BL, Leipsic J, Gaur S, et al. Diagnostic performance of noninvasive fractional flow reserve derived from coronary computed tomography angiography in suspected coronary artery disease: the NXT trial (Analysis of Coronary Blood Flow Using CT Angiography: Next Steps). J Am Coll Cardiol 2014;63(12):1145-1155.

4. Ku DN. Blood flow in arteries. Annu Rev Fluid Mech 1997;29(1):399-434.

5. Resnick N, Yahav H, Shay-Salit A, et al. Fluid shear stress and the vascular endothelium: for better and for worse. Prog Biophys Mol Biol 2003;81(3):177-199.

6. Slager CJ, Wentzel JJ, Gijsen FJH, et al. The role of shear stress in the generation of rupture-prone vulnerable plaques. Nat Clin Pract Cardiovasc Med 2005;2(8):401-407.

7. Vita JA, Treasure CB, Ganz P, Cox DA, Fish RD, Selwyn AP. Control of shear stress in the epicardial coronary arteries of humans: impairment by atherosclerosis. J Am Coll Cardiol 1989;14(5):1193-1199.

8. Han D, Starikov A, Ó Hartaigh B, et al. Relationship between endothelial wall shear stress and high-risk atherosclerotic plaque characteristics for identification of coronary lesions that cause ischemia: A direct comparison with fractional flow reserve. J Am Heart Assoc 2016;5(12):e004186.

9. Neglia D, Rovai D, Caselli C, et al. Detection of significant coronary artery disease by noninvasive anatomical and functional imaging. Circ Cardiovasc Imaging 2015;8(3):1-10.

10. Anagnostopoulos CD, Siogkas PK, Liga R, et al. Characterization of functionally significant coronary artery disease by a coronary computed tomography angiography-based index: a comparison with positron emission tomography. Eur Heart J Cardiovasc Imaging 2019;20(8):897-905.

11. Athanasiou L, Rigas G, Sakellarios AI, et al. Three-dimensional reconstruction of coronary arteries and plaque morphology using CT angiography--comparison and registration with IVUS. BMC Med Imaging 2016;16(1):9. 
12. Siogkas PK, Papafaklis MI, Sakellarios AI, et al. Patient-specific simulation of coronary artery pressure measurements: an in vivo three-dimensional validation study in humans. BioMed Res Int 2015;2015:628416.

13. Sakellarios AI, Papafaklis MI, Siogkas P, et al. Patient-specific computational modeling of subendothelial LDL accumulation in a stenosed right coronary artery: effect of hemodynamic and biological factors. Am J Physiol Heart Circ Physiol 2013;304(11):H1455-H1470.

14. Papafaklis MI, Muramatsu T, Ishibashi Y, et al. Fast virtual functional assessment of intermediate coronary lesions using routine angiographic data and blood flow simulation in humans: comparison with pressure wire - fractional flow reserve. EuroIntervention 2014;10(5):574-583.

15. Sakellarios AI, Rigas G, Kigka V, et al. SMARTool: A tool for clinical decision support for the management of patients with coronary artery disease based on modeling of atherosclerotic plaque process. In: 2017 39th Annual International Conference of the IEEE Engineering in Medicine and Biology Society (EMBC), Jeju, South Korea, July 11-15, 2017. Piscataway, NJ: IEEE, 2017; 96-97.

16. Chang HJ, Lin FY, Lee SE, et al. Coronary Atherosclerotic Precursors of Acute Coronary Syndromes. J Am Coll Cardiol 2018;71(22):2511-2522.

17. Ihdayhid AR, Goeller M, Dey D, et al. Comparison of Coronary Atherosclerotic Plaque Burden and Composition as Assessed on Coronary Computed Tomography Angiography in East Asian and European-Origin Caucasians. Am J Cardiol 2019;124(7):1012-1019.

18. Berti V, Sciagrà R, Neglia D, et al. Segmental quantitative myocardial perfusion with PET for the detection of significant coronary artery disease in patients with stable angina. Eur J Nucl Med Mol Imaging 2016;43(8):15221529.

19. Liga R, Vontobel J, Rovai D, et al. Multicentre multi-device hybrid imaging study of coronary artery disease: results from the EValuation of INtegrated Cardiac Imaging for the Detection and Characterization of Ischaemic Heart Disease (EVINCI) hybrid imaging population. Eur Heart J Cardiovasc Imaging 2016;17(9):951-960.

20. Danad I, Uusitalo V, Kero T, et al. Quantitative assessment of myocardial perfusion in the detection of significant coronary artery disease: cutoff values and diagnostic accuracy of quantitative [(15)O]H2O PET imaging. J Am Coll Cardiol 2014;64(14):1464-1475.

21. Malek AM, Alper SL, Izumo S. Hemodynamic shear stress and its role in atherosclerosis. JAMA 1999;282(21):2035-2042.

22. Park JB, Choi G, Chun EJ, et al. Computational fluid dynamic measures of wall shear stress are related to coronary lesion characteristics. Heart 2016;102(20):1655-1661.

23. Kumar A, Thompson EW, Lefieux A, et al. High Coronary Shear Stress in Patients With Coronary Artery Disease Predicts Myocardial Infarction. J Am Coll Cardiol 2018;72(16):1926-1935.
24. Koskinas KC, Chatzizisis YS, Baker AB, Edelman ER, Stone PH, Feldman $\mathrm{CL}$. The role of low endothelial shear stress in the conversion of atherosclerotic lesions from stable to unstable plaque. Curr Opin Cardiol 2009; 24(6):580-590.

25. Chatzizisis YS, Jonas M, Coskun AU, et al. Prediction of the localization of high-risk coronary atherosclerotic plaques on the basis of low endothelia shear stress: an intravascular ultrasound and histopathology natural history study. Circulation 2008;117(8):993-1002.

26. Eshtehardi P, McDaniel MC, Suo J, et al. Association of coronary wall shear stress with atherosclerotic plaque burden, composition, and distribution in patients with coronary artery disease. J Am Heart Assoc 2012;1(4):e002543.

27. Samady H, Eshtehardi P, McDaniel MC, et al. Coronary artery wall shear stress is associated with progression and transformation of atherosclerotic plaque and arterial remodeling in patients with coronary artery disease. Circulation 2011;124(7):779-788.

28. dela Paz NG, Walshe TE, Leach LL, Saint-Geniez M, D’Amore PA. Role of shear-stress-induced VEGF expression in endothelial cell survival. J Cell Sci 2012;125(Pt 4):831-843.

29. Thondapu V, Bourantas CV, Foin N, Jang IK, Serruys PW, Barlis P. Biomechanical stress in coronary atherosclerosis: emerging insights from computational modelling. Eur Heart J 2017;38(2):81-92.

30. Wentzel JJ, Chatzizisis YS, Gijsen FJH, Giannoglou GD, Feldman CL, Stone PH. Endothelial shear stress in the evolution of coronary atherosclerotic plaque and vascular remodelling: current understanding and remaining questions. Cardiovasc Res 2012;96(2):234-243.

31. Gijsen F, Katagiri Y, Barlis P, et al. Expert recommendations on the assessment of wall shear stress in human coronary arteries: existing methodologies, technical considerations, and clinical applications. Eur Heart J 2019; 40(41):3421-3433.

32. Tremblay JC, Pyke KE. Flow-mediated dilation stimulated by sustained increases in shear stress: a useful tool for assessing endothelial function in humans? Am J Physiol Heart Circ Physiol 2018;314(3):H508-H520.

33. Baskaran L, Ó Hartaigh B, Schulman-Marcus J, Gransar H, Lin F, Min JK. Dense calcium and lesion-specific ischemia: A comparison of CCTA with fractional flow reserve. Atherosclerosis 2017;260:163-168.

34. Driessen RS, Stuijfzand WJ, Raijmakers PG, et al. Effect of Plaque Burden and Morphology on Myocardial Blood Flow and Fractional Flow Reserve. J Am Coll Cardiol 2018;71(5):499-509.

35. Ahmadi A, Leipsic J, Øvrehus KA, et al. Lesion-Specific and Vessel-Related Determinants of Fractional Flow Reserve Beyond Coronary Artery Stenosis. JACC Cardiovasc Imaging 2018;11(4):521-530. 